CARDIOVASCULAR MEDICINE

\title{
Diagnostic accuracy of $B$ type natriuretic peptide and amino terminal proBNP in the emergency diagnosis of heart failure
}

\author{
T Mueller, A Gegenhuber, W Poelz, M Haltmayer
}

Heart 2005;91:606-612. doi: 10.1136/hrt.2004.037762

See end of article for authors' affiliations

\section{Correspondence to:} Dr Meinhard Haltmayer, Department of Laboratory Medicine, Konventhospital Barmherzige Brueder Seilerstaette 2, A-4021 Linz, Austria: meinhard.haltmayer@ bblinz.at

Accepted 28 July 2004

\begin{abstract}
Objective: To compare head to head the diagnostic accuracy of B type natriuretic peptide (BNP) and the amino terminal fragment of its precursor hormone (NT-proBNP) for congestive heart failure (CHF) in an emergency setting.

Methods: 251 consecutive patients presenting to the emergency department with dyspnoea as a chief complaint were prospectively studied. Patients with acute coronary syndromes were excluded. The diagnosis of CHF was based on the Framingham score for CHF plus echocardiographic evidence of systolic or diastolic dysfunction. Blood concentrations of BNP and NT-proBNP were measured by two commercially available assays (Abbott and Roche methods). The diagnostic accuracies of BNP and NTproBNP were assessed by receiver operating characteristic curve analysis.

Results: Areas under the curve for BNP and NT-proBNP in patients with dyspnoea caused by CHF $(n=137)$ and in patients with dyspnoea attributable to other reasons $(n=114)$ did not differ significantly (area under the curve $0.916 \vee 0.903, p=0.277$, statistical power 94\%). Cut off concentrations with the highest diagnostic accuracy were $295 \mathrm{ng} / \mathrm{l}$ for BNP (sensitivity $80 \%$, specificity $86 \%$, diagnostic accuracy $83 \%$ ) and $825 \mathrm{ng} / \mathrm{l}$ for NT-proBNP (sensitivity $87 \%$, specificity $81 \%$, diagnostic accuracy $84 \%$ ). Evaluation of discordant false classifications at these cut off concentrations showed no advantage for either BNP nor NT-proBNP in the biochemical diagnosis of CHF (17 misclassifications by BNP and 14 by NT-proBNP, $p=0.720$ ). In the population studied, age, sex, and renal function had no impact on the diagnostic utility of both tests when compared by logistic regression models.

Conclusions: BNP and NT-proBNP may be equally useful as an aid in the diagnosis of CHF in short of breath patients presenting to the emergency department.
\end{abstract}

B type natriuretic peptide (BNP) is a cardiac neurohormone that derives from the precursor pre-proBNP, containing 134 amino acids and including a signal peptide of 26 amino acids. proBNP, produced by cleavage of the signal peptide, is further split into BNP, which is considered to be the biologically active hormone, and an inactive amino terminal fragment, NT-proBNP. ${ }^{1}$ Blood measurements of BNP have been shown to be useful in differentiating dyspnoea caused by congestive heart failure (CHF) from dyspnoea related to other causes. ${ }^{2}{ }^{3}$ More recently, the BNP (breathing not properly) study showed the usefulness of determining BNP in the emergency department in establishing or excluding CHF in patients with dyspnoea. ${ }^{45}$ The authors of the BNP study showed that the evaluation of dyspnoea was improved by the addition of BNP testing to clinical judgement in the emergency department. ${ }^{5}$ Another recent publication indicated that measurement of NT-proBNP may also be a diagnostic aid in the verification of CHF in patients with shortness of breath. ${ }^{6}$ However, potential advantages of either BNP or NT-proBNP for the diagnosis of CHF in an emergency setting are not established.

Thus, the present study was designed to compare head to head the diagnostic accuracy of BNP and NT-proBNP with respect to $\mathrm{CHF}$ in patients consulting our emergency department with shortness of breath as a chief complaint. Furthermore, we aimed at assessing appropriate cut off concentrations for this clinical setting by means of two currently developed commercially available assays for BNP and NT-proBNP.

\section{METHODS}

\section{Patient recruitment}

From 1 October 2003 to 12 February 2004, all patients presenting with dyspnoea to the emergency department of St
John of God Hospital Linz, Austria, were eligible for the present prospective clinical evaluation. The inclusion criterion was shortness of breath as a chief complaint at the initial patient examination in our emergency department. Patients with ST elevation myocardial infarction, non-ST elevation myocardial infarction, or acute coronary syndrome troponin positive and trauma patients were excluded. During the initial patient examination in our emergency department a blood sample for the determination of BNP and NT-proBNP had to be collected and analysed within the next four hours. Serum creatinine concentrations were routinely determined by Jaffé's kinetic method on a COBAS Integra 700 analyser (Roche Diagnostics, Germany) on the day of blood collection. Estimated glomerular filtration rate (eGFR) was calculated by the Cockgroft and Gault formula. ${ }^{7}$ The complete clinical evaluation including echocardiography and the classification of patients had to be completed within three days after the initial patient examination. For the attending physicians and cardiologists BNP and NT-proBNP results were available in the medical records only after the patients had been completely clinically evaluated and classified. The study protocol was approved by the local ethics committee in accordance with the Declaration of Helsinki. All study participants gave informed consent. The present evaluation was performed and described according to the criteria

\footnotetext{
Abbreviations: AUC, area under the curve; BNP, B type natriuretic peptide; BNP study, breathing not properly study; $\mathrm{CHF}$, congestive heart failure; CV, coefficient of variance; eGFR, estimated glomerular filtration rate; LVEF, left ventricular ejection fraction; NCCLS, National Committee for Clinical Laboratory Standards; NT-proBNP, amino terminal fragment of proBNP; ROC, receiver operating characteristic; STARD, standards for reporting of diagnostic accuracy
} 
suggested by the standards for reporting of diagnostic accuracy (STARD) initiative. ${ }^{8}$

\section{Clinical evaluation of patients}

Each patient enrolled in the study was thoroughly surveyed by taking a history, physical examination, 12 lead ECG, and chest $\mathrm{X}$ ray at the time of the initial patient examination, as well as sonography of the liver (determination of congestive hepatomegaly or hepatic venous congestion). All study participants underwent subsequent echocardiography within three days after the patients' first examination. Left ventricular ejection fraction (LVEF) was measured by the quantitative two dimensional (biplane Simpson) method. Patients were defined as having systolic dysfunction if their LVEF was $\leqslant 50 \%$. Patients with an LVEF $>50 \%$ were further examined for diastolic dysfunction. Each of these patients underwent pulsed wave Doppler examination of mitral inflow before and during Valsalva manoeuvre. Diastolic dysfunction was categorised according to the progression of diastolic dysfunction as normal; mild (grade 1 ), defined as impaired relaxation without evidence of increased filling pressures; moderate (grade 2), defined as impaired relaxation associated with moderately increased filling pressures or pseudonormal filling; and severe (grades 3 and 4), defined as reversible restrictive or fixed restrictive patterns. In the case of LVEF $>50 \%$ and concomitant atrial fibrillation during echocardiography classification of diastolic dysfunction was not possible due to methodological limitations. ${ }^{9}$

\section{Classification of patients (reference standard)}

One of the investigators (GA), also blinded to the BNP and NT-proBNP results, reviewed all medical records pertaining to the patient after completion of the above described clinical evaluation. Thereafter, the final classification was based on the Framingham criteria for the clinical diagnosis of CHF. ${ }^{10}$ For the diagnosis of CHF both of the following had to be present: Framingham CHF score with two major or one major and two minor criteria; and evidence of systolic or diastolic dysfunction as determined by echocardiography. This procedure is in accordance with the guidelines for the diagnosis of CHF in clinical practice. ${ }^{11}$ For patients with a diagnosis other than CHF, the diagnosis was verified on the basis of other observations (for example, roentgenographic signs of chronic obstructive lung disease, pneumonia, abnormal pulmonary function tests, or follow up results such as response to treatment with nebulisers, corticosteroids, and antibiotics).

\section{BNP and NT-proBNP determination (index tests)}

Blood for measurement of natriuretic peptide concentrations was collected by venepuncture in Vacuette polyethylene terephthalate glycol EDTA tubes (Greiner Bio-One, Kremsmünster, Austria) at the initial patient examination in the emergency department. Blood samples were centrifuged at $3500 \mathrm{~g}$ for 10 minutes at $4^{\circ} \mathrm{C}$ immediately after collection. Both BNP and NT-proBNP were analysed within four hours after blood withdrawal. BNP was assayed on an AxSYM analyser (Abbott Laboratories, Abbott Park, Illinois, USA). The AXSYM BNP assay is a fully automated microparticle enzyme immunoassay with two monoclonal mouse antibodies in a two step sandwich format. ${ }^{12}$ NT-proBNP was measured on an Elecsys 2010 instrument (Roche Diagnostics). The Elecsys NT-proBNP test is a fully automated two site (sandwich) enzyme immunoassay with electrochemiluminescent technology and is designed for the determination of NT-proBNP in human serum and plasma. ${ }^{13}$

The precision of the two methods was evaluated according to the National Committee for Clinical Laboratory Standards (NCCLS) guideline EP5-A. ${ }^{14}$ Three pooled patient plasma samples were aliquoted into 40 tubes of $1.5 \mathrm{ml}$ for each concentration and frozen at $-70^{\circ} \mathrm{C}$. We analysed these samples in duplicate in two runs every day for 20 days on the two analysers. Total imprecision was calculated by the NCCLS double run precision evaluation test. ${ }^{14}$ Precision data of the two methods were as follows: the AxSYM BNP assay had a total coefficient of variance $(\mathrm{CV})$ of $8.1 \%$ at a mean concentration of $108 \mathrm{ng} / \mathrm{l}$ (pool 1), a total CV of $7.5 \%$ at a mean concentration of $524 \mathrm{ng} / \mathrm{l}$ (pool 2), and a total CV of $10 \%$ at a mean concentration of $2117 \mathrm{ng} / \mathrm{l}$ (pool 3). The Elecsys NT-proBNP assay had a total CV of $3.8 \%$ at a mean concentration of $246 \mathrm{ng} / \mathrm{l}$ (pool 1), a total CV of $4.7 \%$ at a mean concentration of $891 \mathrm{ng} / \mathrm{l}$ (pool 2), and a total CV of $2.2 \%$ at a mean concentration of $10666 \mathrm{ng} / \mathrm{l}$ (pool 3).

\section{Statistical analysis}

Univariate data on demographic and clinical features were compared between patients with dyspnoea caused by CHF and patients with dyspnoea attributable to other reasons by the non-parametric Mann-Whitney U test or Fisher's exact test as appropriate ( $p$ values were not adjusted for multiple comparisons and are therefore only descriptive). To determine the diagnostic accuracy of BNP and NT-proBNP for CHF, receiver operating characteristic (ROC) plots were analysed and areas under the curve (AUCs) were calculated for both analytes. AUCs were compared according to the method by Hanley and McNeil..$^{15}$ Cut off concentrations for BNP and NT-proBNP were determined at the $90 \%$ and $95 \%$ sensitivity criterion derived directly from the ROC curves. Furthermore, cut off concentrations with the highest diagnostic accuracy (that is, optimal cut off concentrations, defined as points of the ROC curves, in which the sum of false negative and false positive results was lowest) and the approved cut points were evaluated. Positive and negative predictive values at these cut off concentrations were calculated by using the ratio of cases in the positive and negative groups (reflecting the prevalence of the disease in our study population). To determine odds ratios (unadjusted and controlling for confounding covariates) for the detection of CHF with respect to BNP and NT-proBNP thresholds of highest diagnostic accuracy, logistic regression analysis without variable selection technique was performed. Dichotomous variables were coded with an indicator variable of 1 for having the disease and 0 for its absence. The diagnostic accuracies of both assays at optimal cut off concentrations as determined by ROC analysis were compared by the McNemar test (comparison of discordant pairs of false biochemical classifications). Spearman's coefficient of rank correlation was used to asses the relation of BNP and NT-proBNP concentrations in the study population. Data were statistically analysed with SPSS 10.0 software (SPSS Inc, Chicago, Illinois, USA), the MedCalc 7.2.1.0 package (MedCalc Software, Mariakerke, Belgium), and the software N (IDV, Gauting, Germany). All probabilities were two tailed and $p<0.05$ was regarded as significant. Sample size calculation for the present study was based on the following assumptions: AUC for BNP approximately 0.900 with an estimated SE of 0.030 and a correlation of BNP and NTproBNP concentrations of 0.900 . Thus, to detect a difference of 5\% in AUCs for the two analytes, our calculation showed that a sample size of 250 patients had to be enrolled into this study $(\alpha=0.050$ two sided, $\beta=0.100)$.

\section{RESULTS}

Of 293 patients referred with dyspnoea, 276 met the primary inclusion criteria and were enrolled. For 11 patients the clinical evaluation (Framingham CHF classification or echocardiography) could not be completed within three days. Thus, 265 patients with complete clinical evaluation were 
293 presenting to the emergency department with dyspnoea as a chief complaint

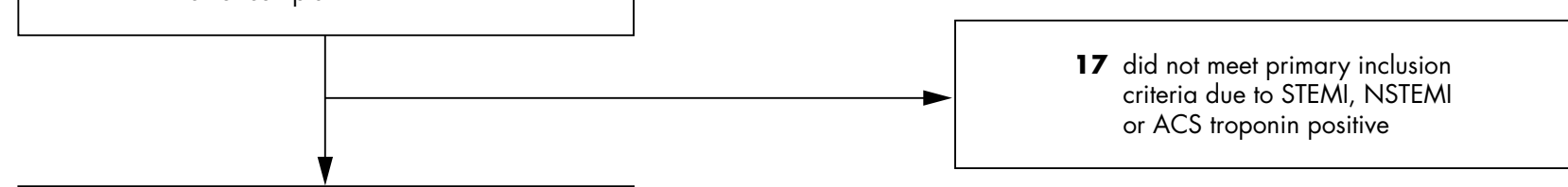

276 met primary inclusion criteria

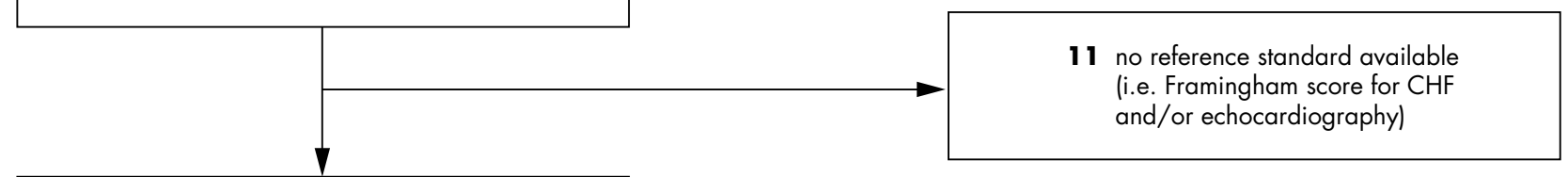

265 with reference standard were allocated for CHF classification

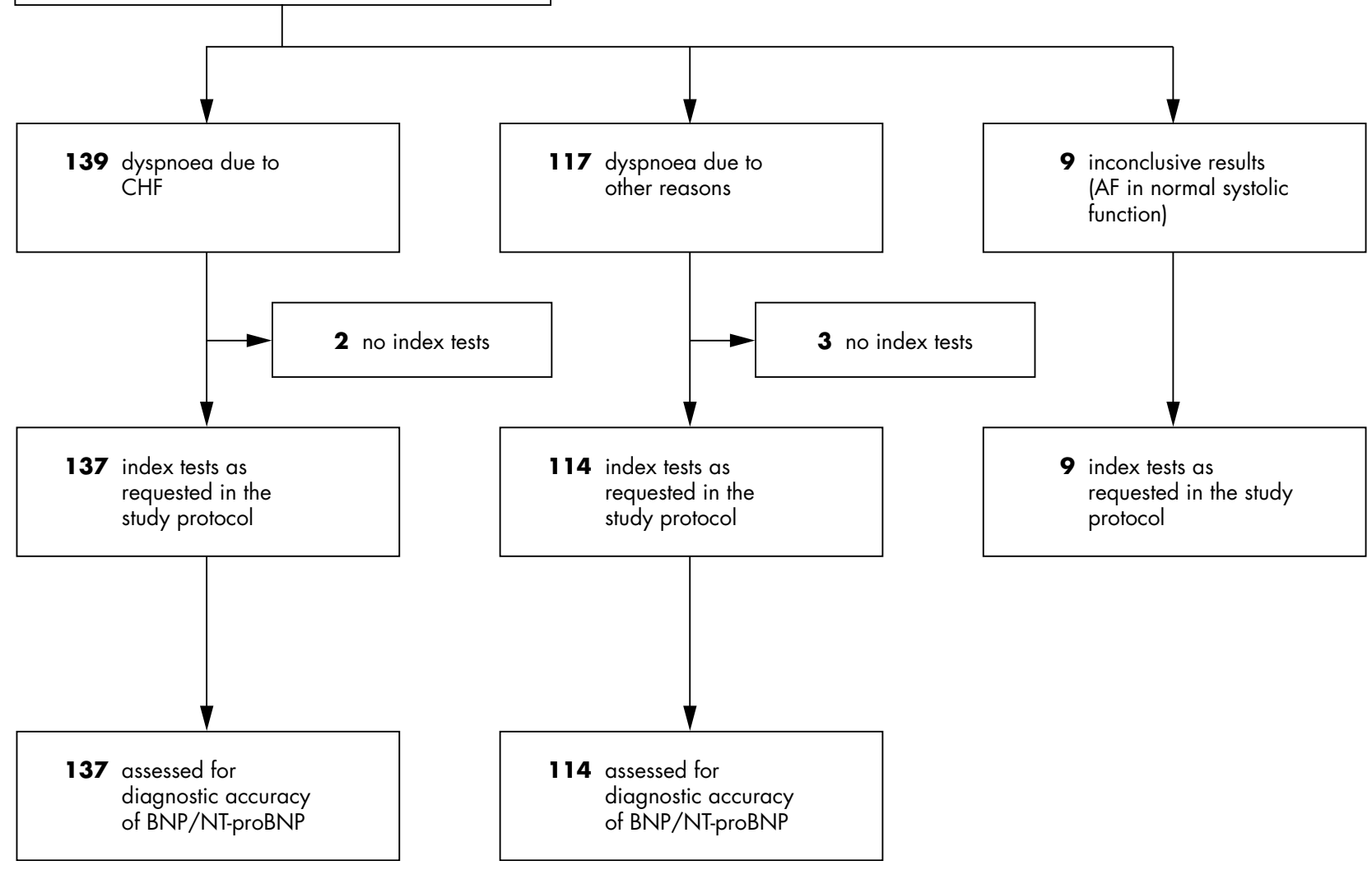

Figure 1 Trial profile. ACS, acute coronary syndromes; AF, atrial fibrillation; BNP, B-type natriuretic peptide; CHF, congestive heart failure; NSTEMI, non-ST elevation myocardial infarction; NT-proBNP, amino terminal fragment of proBNP; STEMI, ST elevation myocardial infarction.

allocated to CHF classification. In nine patients with LVEF $>50 \%$ concomitant atrial fibrillation during echocardiography prohibited an exact classification of diastolic dysfunction. Of the remaining 256 patients, five were excluded because both index tests (BNP and NT-proBNP) were not completed within four hours after blood withdrawal. Finally, 137 patients with dyspnoea due to CHF were compared with 114 patients with dyspnoea attributable to other reasons in the present study. Figure 1 provides comprehensive information. Patients with end stage renal disease on dialysis were not enrolled into the present study, although this condition was not an exclusion criterion.
Table 1 lists the demographic and clinical characteristics of the study participants $(n=251)$. Figure 2 presents box and whisker plots for BNP and NT-proBNP in both study groups. Of the overall study group $(n=251), 100 \%$ had dyspnoea on exertion, $16 \%$ had orthopnoea, $30 \%$ had paroxysmal nocturnal dyspnoea, 29\% had nocturnal cough, 17\% had jugular venous distension, $21 \%$ had pulmonary rales, $10 \%$ had a third heart sound, and $21 \%$ had oedema of the legs or feet. As mentioned, 137 met the criteria for dyspnoea caused by $\mathrm{CHF}$, and 114 patients attended our emergency department because of dyspnoea attributable to other causes, including 10 patients with a history of CHF who were 
Table 1 Patient characteristics according to CHF classification $(n=251)$

\begin{tabular}{|c|c|c|c|}
\hline \multirow[b]{2}{*}{ Demographic and clinical features } & \multicolumn{2}{|l|}{ Cause of dyspnoea } & \multirow[b]{2}{*}{$p$ Value $^{*}$} \\
\hline & CHF $(n=137)$ & Other $(n=114)$ & \\
\hline Men/women & $128 / 9$ & $106 / 8$ & 1.000 \\
\hline Age (years) & $76(69-82)$ & $69(58-78)$ & $<0.001$ \\
\hline $\mathrm{BMI}\left(\mathrm{kg} / \mathrm{m}^{2}\right)$ & $26(24-29)$ & $26(23-30)$ & 0.628 \\
\hline Arterial hypertension & 86 & 55 & 0.022 \\
\hline Diabetes mellitus & 39 & 19 & 0.035 \\
\hline Renal dysfunction $\dagger$ & 53 & 21 & $<0.001$ \\
\hline AF & 65 & 18 & $<0.001$ \\
\hline Known CAD & 81 & 36 & $<0.001$ \\
\hline History of $\mathrm{CHF}$ & 65 & 10 & $<0.001$ \\
\hline \multicolumn{4}{|l|}{ Symptoms of CHF } \\
\hline Dyspnoea on exertion & 137 & 114 & NA \\
\hline Órthopnoea & 28 & 11 & 0.023 \\
\hline PND & 53 & 22 & 0.001 \\
\hline Nocturnal cough & 50 & 22 & 0.003 \\
\hline \multicolumn{4}{|l|}{ Signs of $\mathrm{CHF}$} \\
\hline JVD & 38 & 4 & $<0.001$ \\
\hline Pulmonary rales & 44 & 8 & $<0.001$ \\
\hline Third heart sound & 22 & 2 & $<0.001$ \\
\hline Peripheral oedema & 40 & 13 & 0.001 \\
\hline \multicolumn{4}{|l|}{ Framingham score } \\
\hline Classification positive & 137 & 7 & - \\
\hline Major criteria (points) & $2(1-3)$ & $0(0-1)$ & - \\
\hline Minor criteria (points) & $2(1-3)$ & $1(1-2)$ & - \\
\hline \multicolumn{4}{|l|}{ NYHA class } \\
\hline 1 & 0 & 10 & NA \\
\hline$\|$ & 59 & 0 & NA \\
\hline III & 53 & 0 & NA \\
\hline IV & 25 & 0 & NA \\
\hline \multicolumn{4}{|l|}{ Echocardiographic data } \\
\hline $\operatorname{LVEF}(\%)$ & $40(28-48)$ & $59(55-62)$ & $<0.001$ \\
\hline Normal function & 0 & 43 & \\
\hline Diastolic dysfunction & 21 & 53 & NA \\
\hline Systolic dysfunction & 116 & 18 & NA \\
\hline \multicolumn{4}{|l|}{ Biochemical markers } \\
\hline Serum creatinine $(\mu \mathrm{mol} / \mathrm{l})$ & $80(62-115)$ & $71(62-88)$ & 0.006 \\
\hline eGFR $(\mathrm{ml} / \mathrm{min})$ & $69(49-101)$ & $95(66-142)$ & $<0.001$ \\
\hline BNP (ng/l) & $792(363-1714)$ & $65(10-196)$ & $<0.001$ \\
\hline NT-proBNP (ng/l) & 3275 (1398-6979) & $248(77-729)$ & $<0.001$ \\
\hline \multicolumn{4}{|c|}{$\begin{array}{l}\text { Data are presented as median (25th-75th centiles) or number. } \\
\text { *Non-parametric Mann-Whitney U test or Fisher's exact test as appropriate; tdefined as estimated glomerular } \\
\text { filtration rate (eGFR) <60 ml/min. } \\
\text { AF, atrial fibrillation (first episode, paroxysmal, persistent, or permanent); BMI, body mass index; BNP, B type } \\
\text { natriuretic peptide; CAD, coronary artery disease; CHF, congestive heart failure; JVD, jugular venous distension } \\
\text { LVEF, left ventricular ejection fraction; NA, not applicable; NT-proBNP, amino terminal fragment of proBNP; } \\
\text { NYHA, New York Heart Association; PND, paroxysmal nocturnal dyspnoea. }\end{array}$} \\
\hline
\end{tabular}

asymptomatic for this disorder (NYHA class I). Reasons for dyspnoea in these 114 patients were chronic obstructive pulmonary disease $(n=41)$, pneumonia $(n=22)$, bronchitis/asthma $(n=12)$, malignancy of the lung $(n=6)$, musculoskeletal chest pain $(n=6)$, hypertension $(n=6)$, acute coronary syndrome troponin negative $(\mathrm{n}=6)$, tachycardia/arrhythmia $(\mathrm{n}=5)$, and other causes $(\mathrm{n}=11)$ such as pulmonary embolism, interstitial lung disease, anaemia, or pericardial disease.

In distinguishing between patients with dyspnoea caused by CHF $(\mathrm{n}=137)$ and patients with dyspnoea attributable to other causes $(\mathrm{n}=114)$, the AUCs were 0.916 ( $\mathrm{SE}=0.018,95 \%$ confidence interval $(\mathrm{CI}) 0.874$ to 0.947 ) for BNP, and 0.903 ( $\mathrm{SE}=0.019,95 \%$ CI 0.859 to 0.939 ) for NT-proBNP (fig 3). Comparison of the ROC curves showed no significant difference between the AUCs for BNP and NTproBNP (difference of AUCs 0.013, SE $=0.012,95 \% \mathrm{CI}$ -0.011 to $0.037, \mathrm{p}=0.277$ ); a power calculation showed that the power of this analysis was $94 \%$.

Table 2 gives detailed diagnostic information including the appropriate decision statistics for the biochemical diagnosis of clinical CHF. Using the cut off concentrations with the highest diagnostic accuracy, classification by both BNP and NT-proBNP was correct in 194 patients and incorrect in 26 patients. Comparing the 17 misclassifications by the BNP assay to the 14 by the NT-proBNP assay with the McNemar test, the difference was not significant ( $p=0.720)$ (table 3 ).

Seven patients with normal systolic and diastolic dysfunction were classified as false positive by means of the Framingham criteria for the clinical diagnosis of CHF as table 1 indicates. In six of these patients BNP and NT-proBNP concentrations were low, so these two analytes would have contributed to a correct diagnosis of non-CHF. Only one of these seven patients had increased BNP and NT-proBNP plasma concentrations, which was attributable to a pulmonary embolism.

Univariate odds ratios for the detection of CHF were calculated with BNP or NT-proBNP as independent variables dichotomised according to the above determined thresholds. In addition, odds ratios for CHF with respect to BNP and NTproBNP adjusted for age, sex, and eGFR were determined (adjusted regression model l) to estimate the influence of these potential confounders. We further included the history of CHF as well as clinical variables (signs and symptoms of $\mathrm{CHF}$ ) in another analysis (adjusted regression model 2) to determine the impact of these covariates on the predictive value of the two analytes for CHF. Table 4 specifies results of these analyses. When calculating a logistic regression with CHF as the dependent variable and age, sex, eGFR, history of CHF, and the presence of orthopnoea, paroxysmal nocturnal 


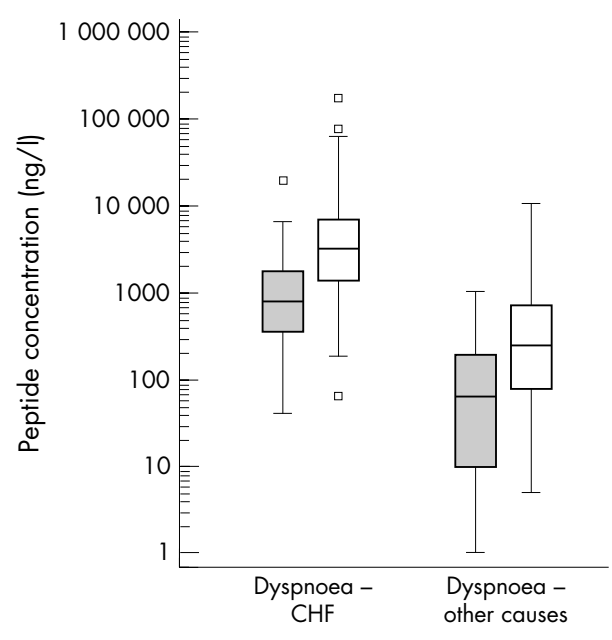

Figure 2 Box and whisker plots of BNP and NT-proBNP for patients with dyspnoea caused by CHF ( $\mathrm{n}=137)$ and for patients with dyspnoea attributable to non-cardiac causes $(n=114)$. In the box and whisker plots, the central box spans from the lower to the upper quartile, the middle line is the median, the whiskers extend from the minimum to the maximum concentration, excluding outside and far out concentrations, which are displayed as separate points. Grey boxes, box and whisker plots for BNP; white boxes, box and whisker plots for NTproBNP.

dyspnoea, nocturnal cough, jugular venous distension, pulmonary rales, third heart sound, and peripheral oedema as independent variables (excluding BNP and NT-proBNP), we found a diagnostic accuracy of $79 \%$ for this statistical model.

Figure 4 shows a scatter plot for BNP versus NT-proBNP concentrations in the study population. The range for this analyte comparison was limited to the reportable ranges of the two assays (that is, $4000 \mathrm{ng} / \mathrm{l}$ for AxSYM BNP and $35000 \mathrm{ng} / \mathrm{l}$ for Elecsys NT-proBNP) to avoid any dilution. Thus, from the 251 patients investigated in the clinical part of the study, only 239 samples were eligible for the correlation analysis because 12 samples had BNP or NT-proBNP concentrations above the measurement ranges of one of the assays. Spearman's coefficient of rank correlation was 0.914 (95\% CI 0.890 to $0.932, \mathrm{p}<0.001$ ).

\section{DISCUSSION}

BNP measurements have been shown to add important information to clinical judgement in establishing a final diagnosis of CHF. ${ }^{5}$ Since our evaluation produced similar AUCs and comparable sensitivity, specificity, and diagnostic accuracy at selected cut off concentrations for BNP and

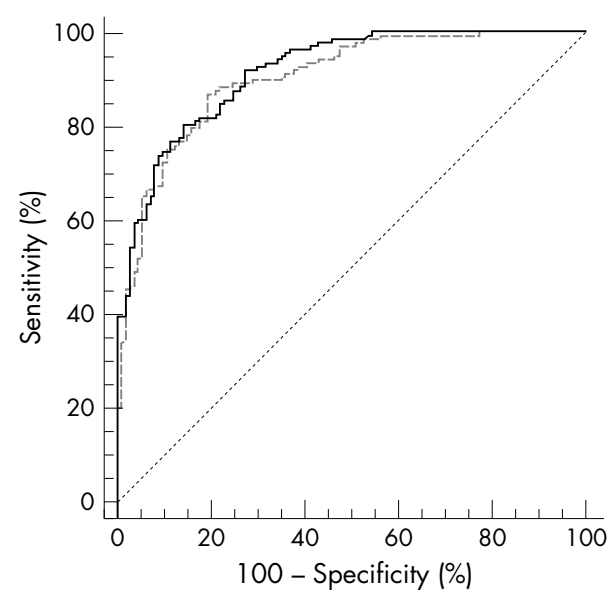

Figure 3 Receiver operating characteristic (ROC) plot for BNP and NTproBNP comparing patients with dyspnoea caused by CHF $(n=137)$ versus patients with dyspnoea attributable to other reasons ( $n=114$ ). The area under the curve for BNP is 0.916 and for NT-proBNP is 0.903 (difference of 0.013 not significant, $p=0.277$ ). Solid line, ROC curve for BNP; dashed line, ROC curve for NT-proBNP.

NT-proBNP in the biochemical diagnosis of CHF, NT-proBNP measurements should also improve the emergency evaluation of dyspnoeic patients. In addition, comparison of discordant false classifications on the basis of optimal cut off concentrations for BNP and NT-proBNP underlined the similar diagnostic utility of the two analytes. Thus, our findings indicate that BNP and NT-proBNP may be equally useful as an aid for the diagnosis of CHF in patients consulting an emergency department with shortness of breath as a chief complaint. Of course, different cut off concentrations have to be considered for the analytes BNP and NT-proBNP as table 2 shows. This is a consequence of a slower plasma clearance of NT-proBNP than of the biologically active peptide BNP resulting in higher circulating concentrations of NTproBNP, although both peptides are released by cardiomyocytes on an equimolar basis. ${ }^{1}$ Furthermore, the different mechanisms of plasma clearance (neutral endopeptidase clearance receptors for BNP versus renal clearance for NTproBNP) ${ }^{1}$ result in an only moderate correlation of plasma concentrations of the two analytes (fig 4).

As shown by logistic regression analysis, we observed no relevant influences of age, sex, and renal function (eGFR) on the diagnostic value of BNP and NT-proBNP in the present study. The odds ratios of BNP and NT-proBNP for the prediction of CHF did not change when calculating the model unadjusted for potential confounders and the model controlling for age, sex, and eGFR. In addition, the overall accuracy

Table 2 Diagnostic information for the biochemical diagnosis of $\mathrm{CHF}$

\begin{tabular}{lllllll}
\hline Analyte & $\begin{array}{l}\text { Cut off } \\
\text { concentration (ng/l) }\end{array}$ & $\begin{array}{l}\text { Sensitivity } \\
(\mathbf{9 5 \%} \mathbf{C l})\end{array}$ & $\begin{array}{l}\text { Specificity } \\
(\mathbf{9 5 \%} \mathrm{Cl})\end{array}$ & PPV & NPV & $\begin{array}{l}\text { Diagnostic } \\
\text { accuracy }\end{array}$ \\
\hline BNP & $100^{*}$ & $96 \%(92$ to 99$)$ & $61 \%(52$ to 70$)$ & 0.75 & 0.93 & $80 \%$ \\
& 118 & $95 \%(90$ to 98$)$ & $64 \%(55$ to 73$)$ & 0.76 & 0.91 & $81 \%$ \\
& 160 & $90 \%(84$ to 95$)$ & $73 \%(64$ to 81$)$ & 0.80 & 0.87 & $83 \%$ \\
NT-proBNP & 295 292 & $80 \%(73$ to 87$)$ & $86 \%(78$ to 92$)$ & 0.87 & 0.78 & $83 \%$ \\
& $125 / 450^{*}$ & $95 \%(90$ to 98$)$ & $53 \%(43$ to 62$)$ & 0.71 & 0.90 & $76 \%$ \\
& 476 & $94 \%(89$ to 97$)$ & $46 \%(37$ to 56$)$ & 0.68 & 0.87 & $73 \%$ \\
& $825+$ & $90 \%(84$ to 95$)$ & $65 \%(55$ to 74$)$ & 0.76 & 0.85 & $79 \%$ \\
& & $87 \%(80$ to 92$)$ & $81 \%(72$ to 88$)$ & 0.84 & 0.84 & $84 \%$ \\
\hline
\end{tabular}

*Approved cut off concentrations for the AxSYM BNP assay (100 ng/l) and Elecsys NT-proBNP assay (dual cut off $125 \mathrm{ng} / \mathrm{l}$ for patients $<75$ years of age and $450 \mathrm{ng} / \mathrm{l}$ for those $\geqslant 75$ years of age); tcriterion concentrations corresponding to the highest diagnostic accuracy (that is, optimal cut off concentrations with minimum false negative and false positive results).

$\mathrm{Cl}$, confidence interval; NPV, negative predictive value; PPV, positive predictive value. 
Table 3 Results of biochemical classification of patients with and without $\mathrm{CHF}$ at cut off concentrations with the highest diagnostic accuracy for BNP and NT-proBNP*

\begin{tabular}{lllr}
\hline & \multicolumn{2}{l}{ BNP } & \\
\cline { 2 - 3 } NT-proBNP & $\begin{array}{l}\text { False } \\
\text { classification }\end{array}$ & $\begin{array}{l}\text { Correct } \\
\text { classification }\end{array}$ & Total \\
\hline False classification & 26 & 14 & 40 \\
Correct classification & 17 & 194 & 211 \\
Total & 43 & 208 & 251 \\
\hline
\end{tabular}

*295 ng/l for BNP and $825 \mathrm{ng} / \mathrm{l}$ for NT-proBNP. McNemar test $p=0.720$.

of the two logistic regression models was equal (that is, $83 \%$ and $84 \%$ for the models with BNP and NT-proBNP, respectively), indicating that age, sex, and eGFR did not add any relevant diagnostic information (despite significant univariate differences between the study groups in terms of age and eGFR). Although previous studies described an association between natriuretic peptide concentrations and age, sex, and renal function, in particular in patients with cardiac impairment, ${ }^{16-18}$ these confounders obviously had no impact on the diagnostic utility of BNP and NT-proBNP in the present study. BNP and NT-proBNP concentrations are known to increase with age, female sex, and renal dysfunction, which may not be sufficient to modify the diagnostic performance of BNP and NT-proBNP in an emergency setting. However, regarding renal function, our statement is restricted to eGFR and we must mention that the median of eGFRs was $84 \mathrm{ml} / \mathrm{min}$ (25th-75th centile $56-119 \mathrm{ml} / \mathrm{min}$ ) in the entire study population, with 74 patients having an eGFR $<60 \mathrm{ml} / \mathrm{min}$.

Adding all clinical information available in the emergency department to the above logistic regression model (that is, history of CHF and the presence of orthopnoea, paroxysmal nocturnal dyspnoea, nocturnal cough, jugular venous distension, pulmonary rales, third heart sound, and peripheral oedema) increased the diagnostic accuracy to $87 \%$. Conversely, the odds ratios for BNP and NT-proBNP decreased as expected, indicating the predictive value of the clinical signs and symptoms of CHF. Removing BNP and NTproBNP, respectively, from this model reduced the diagnostic accuracy of the statistical model (including age, sex, eGFR, and the clinical information described above) to $79 \%$. Thus, BNP and NT-proBNP added about $8 \%$ accuracy in the prediction of CHF to the clinical information in an emergency setting. On the other hand, BNP and NT-proBNP alone



Figure 4 Scatter plot for BNP versus NT-proBNP. Spearman's coefficient of rank correlation ( $\mathrm{n}=239)$ : $0.914(95 \% \mathrm{Cl} 0.890$ to $0.932, p<0.001$ ). Since the cumulative sum test for linearity showed a significant deviation from linearity $(p<0.010)$, results of regression analysis (Passing and Bablok) are not given.

appeared to have a greater predictive value for symptomatic CHF in an emergency department (accuracies of $83 \%$ and $84 \%$, respectively) than taking together all the clinical information (accuracy of 79\%).

In the present study the reported decision statistics were mainly related to cut off concentrations with the highest diagnostic accuracy, defined as points of the ROC curves in which the sum of false negative and false positive results is lowest indicating the points of the curves nearest the top left hand corner. Therefore, these points give an idea of the shape of the ROC curve having mainly a mathematical relevance. However, for clinical practice we recommend referring to cut off concentrations with $90 \%$ or $95 \%$ sensitivity for both BNP and NT-proBNP. These cut off concentrations can be used to rule out a patient with a high probability according to the previously reported high negative predictive values of BNP and NT-proBNP in CHF. ${ }^{11}{ }^{19}$ In this context, the currently approved dual cut off concentration for NT-proBNP is not advantageous compared with a single cut point, especially in terms of the overall diagnostic accuracy as table 2 shows.

Compared with other studies in this field the strengths of our trial are the stringent study design reported according to the suggestions of the STARD initiative, ${ }^{8}$ the performance of echocardiography in each study participant within three days

Table 4 Results of logistic regression analysis

\begin{tabular}{|c|c|c|c|c|c|}
\hline \multirow[b]{2}{*}{ Independent variable } & \multirow{2}{*}{$\begin{array}{l}\text { Cut off } \\
\text { concentration } \\
\text { (ng/l) }\end{array}$} & \multirow[b]{2}{*}{$\begin{array}{l}\text { Odds ratio } \\
(95 \% \mathrm{Cl}, \mathrm{p} \text { value) }\end{array}$} & \multirow[b]{2}{*}{$\begin{array}{l}\text { Diagnostic } \\
\text { accuracy* }\end{array}$} & \multicolumn{2}{|c|}{ Incorrect classification } \\
\hline & & & & $\begin{array}{l}\text { False } \\
\text { positive }\end{array}$ & $\begin{array}{l}\text { False } \\
\text { negative }\end{array}$ \\
\hline \multicolumn{6}{|c|}{ Prediction of symptomatic $\mathrm{CHF}$, unadjusted model } \\
\hline BNP & 295 & $25(13$ to $49,<0.001)$ & $83 \%$ & $n=16$ & $\mathrm{n}=27$ \\
\hline NT-proBNP & 825 & $28(14$ to $55,<0.001)$ & $84 \%$ & $\mathrm{n}=22$ & $\mathrm{n}=18$ \\
\hline \multicolumn{6}{|c|}{ Prediction of symptomatic CHF, adjusted model 1 (controlling for age, sex, and eGFR) } \\
\hline BNP & 295 & $27(13$ to $56,<0.001)$ & $83 \%$ & $n=16$ & $\mathrm{n}=27$ \\
\hline NT-proBNP & 825 & $29(14$ to $61,<0.001)$ & $84 \%$ & $\mathrm{n}=22$ & $\mathrm{n}=18$ \\
\hline \multicolumn{6}{|c|}{$\begin{array}{l}\text { Prediction of symptomatic CHF, adjusted model } 2 \text { (controlling for age, sex, eGFR, history of CHF, and the presence } \\
\text { of orthopnoea, PND, nocturnal cough, JVD, pulmonary rales, third heart sound, and peripheral oedema) }\end{array}$} \\
\hline BNP & 295 & $10(4$ to $24,<0.001)$ & $87 \%$ & $n=16$ & $n=18$ \\
\hline NT-proBNP & 825 & $13(6$ to $32,<0.001)$ & $87 \%$ & $n=19$ & $n=15$ \\
\hline \multicolumn{6}{|c|}{$\begin{array}{l}\text { *Diagnostic accuracy of the statistical model including all the given covariates simultaneously. } \\
\text { Odds ratios }(95 \% \mathrm{Cl}, \mathrm{p} \text { value) are related to the two analytes. Diagnostic accuracy and false positive and false } \\
\text { negative results of classification are derived from the respective whole statistical model. Observed frequencies: } \\
\text { patients with dyspnoea caused by } \mathrm{CHF}, \mathrm{n}=137 \text {; patients with dyspnoea attributable to other causes, } \mathrm{n}=114 \text {. }\end{array}$} \\
\hline
\end{tabular}


after their enrolment (assessing both systolic and diastolic dysfunction), and the determination of both index tests (BNP and NT-proBNP) directly after blood collection at the initial examination in our emergency department (the stability of these analytes in frozen plasma samples is controversial). However, one limitation of the present study is the preponderance of men. In this context, we must point out that our hospital is traditionally mainly responsible for medical care of male patients in the extended area of Linz, Austria. Another limitation of the present trial may be related to the determination of diastolic dysfunction. We did not use Doppler tissue imaging of mitral annular motion in this study as one of the examinations applied for the assessment of diastolic dysfunction'. Nevertheless, this is the first time that diastolic dysfunction has been determined in a study evaluating the diagnostic accuracy of BNP and NT-proBNP in an emergency setting and, thus, the chosen approach, which is clinically useful, is valid in our opinion.

In conclusion, this study showed that BNP and NT-proBNP may be equally useful as an aid in the diagnosis of CHF in patients presenting to the emergency department with shortness of breath as a chief complaint. We observed no relevant influences of age, sex, and renal function (eGFR) on the diagnostic value of the two analytes. BNP and NTproBNP alone had a greater predictive value for symptomatic CHF than the clinical information available in the emergency department.

\section{ACKNOWLEDGEMENTS}

This work was supported in part by a grant for scientific research from the Upper Austrian Government. We thank Abbott Diagnostics (Vienna, Austria) and Roche Diagnostics (Vienna, Austria) for providing reagents free of charge. Neither of these companies played a part in (1) the design of the study, (2) data collection, analysis, and interpretation, and (3) preparation of the manuscript.

\section{Authors' affiliations}

T Mueller, M Haltmayer, Department of Laboratory Medicine, Konventhospital Barmherzige Brueder, Linz, Austria

A Gegenhuber, Department of Internal Medicine, Konventhospital Barmherzige Brueder

W Poelz, Institute for Applied System Sciences and Statistics, University of Linz

\section{REFERENCES}

1 McCullough PA Omland T, Maisel AS. B-type natriuretic peptides: a diagnostic breakthrough for clinicians. Rev Cardiovasc Med 2003;4:72-80.

2 Davis M, Espiner E, Richards G, et al. Plasma brain natriuretic peptide in assessment of acute dyspnoea. Lancet 1994;343:440-4.

3 Dao Q, Krishnaswamy P, Kazanegra R, et al. Utility of B-type natriuretic peptide in the diagnosis of congestive heart failure in an urgent-care setting. $J$ Am Coll Cardiol 2001;37:379-85.

4 Maisel AS, Krishnaswamy P, Nowak RM, et al. Rapid measurement of B-type natriuretic peptide in the emergency diagnosis of heart failure. N Engl J Med 2002;347:161-7.

5 McCullough PA, Nowak RM, McCord J, et al. B-type natriuretic peptide and clinical judgment in emergency diagnosis of heart failure: analysis from breathing not properly (BNP) multinational study. Circulation 2002;106:416-22.

6 Lainchbury JG, Campbell E, Frampton CM, et al. Brain natriuretic peptide and $\mathrm{n}$-terminal brain natriuretic peptide in the diagnosis of heart failure in patients with acute shortness of breath. J Am Coll Cardiol 2003;42:728-35.

7 Cockgroft DW, Gault MH. Prediction of creatinine clearance from serum creatinine. Nephron 1976;16:31-41.

8 Bossuyt PM, Reitsma JB, Bruns DE, et al. Towards complete and accurate reporting of studies of diagnostic accuracy: the STARD initiative. Standards for reporting of diagnostic accuracy. Clin Chem 2003;49:1-6.

9 Redfield MM, Jacobsen SJ, Burnett JC Jr, et al. Burden of systolic and diastolic ventricular dysfunction in the community: appreciating the scope of the heart failure epidemic. JAMA 2003;289:194-202.

10 Senni M, Tribouilloy CM, Rodeheffer RJ, et al. Congestive heart failure in the community: a study of all incident cases in Olmsted County, Minnesota, in 1991. Circulation 1998;98:2282-9.

11 Remme WJ, Swedberg K. Comprehensive guidelines for the diagnosis and treatment of chronic heart failure. Task force for the diagnosis and treatment of chronic heart failure of the European Society of Cardiology. Eur J Heart Fail 2002;4:11-22.

12 Mueller T, Gegenhuber A, Poelz W, et al. Preliminary evaluation of the AxSYM B-type natriuretic peptide (BNP) assay and comparison with the ADVIA Centaur BNP assay. Clin Chem 2004;50:1 104-6.

13 Mueller T, Gegenhuber A, Poelz W, et al. Comparison of the Biomedica NTproBNP enzyme immunoassay and the Roche NT-proBNP chemiluminescence immunoassay: implications for the prediction of symptomatic and asymptomatic structural heart disease. Clin Chem 2003:49:976-9.

14 National Committee for Clinical Laboratory Standards. Evaluation of precision performance of clinical chemistry devices: approved guideline. NCCLS document EP5-A. Wayne: NCCLS, 1999.

15 Hanley JA, McNeil BJ. A method of comparing the areas under receiver operating characteristic curves derived from the same cases. Radiology 1983; 148:839-43

16 Redfield MM, Rodeheffer RJ, Jacobsen SJ, et al. Plasma brain natriuretic peptide concentration: impact of age and gender. J Am Coll Cardiol 2002;40:976-82

17 Raymond I, Groenning BA, Hildebrandt PR, et al. The influence of age, sex and other variables on the plasma level of $\mathrm{N}$-terminal pro brain natriuretic peptide in a large sample of the general population. Heart 2003;89:745-51

18 McCullough PA, Sandberg KR. B-type natriuretic peptide and renal disease. Heart Fail Rev 2003;8:355-8.

19 Clerico A, Emdin M. Diagnostic accuracy and prognostic relevance of the measurement of cardiac natriuretic peptides: a review. Clin Chem 2004;50:33-50.

\section{FROM BMJ JOURNALS}

\section{Excess in cardiovascular events on Mondays: a meta-analysis and prospective study} Adrian G Barnett, Annette J Dobson Please visit the
Heart website [www. heartin]. com] for a link to the full text of this article.

he aim of this paper was to summarise the reported excess in coronary events on Mondays, and examine the evidence for three competing explanations: stress, alcohol consumption, or registration errors. A review of the literature found 28 studies covering 16 countries and over 1.6 million coronary events. The overall Monday excess was small; in a population experiencing 100 coronary events per week there was one more event on Monday than other days. The excess was larger in men and in studies including sudden cardiac death or cardiac arrests. In a prospective study an increase in events on Mondays was associated with greater alcohol consumption, lower rainfall, and the month of January. The excess in coronary events on Mondays is a persistent phenomenon. The size of the effect varies widely between populations. There is some evidence of an association with alcohol consumption, but a definitive explanation remains elusive and is likely to remain so because of the smallness of the effect and the paucity of high quality data.

A Journal of Epidemiology and Community Health 2005;59:109-114. 